\title{
Cardiac magnetic resonance imaging and follow-up of pacemaker events to identify the etiology and natural history of heart blocks
}

Andres E Carmona-Rubio ${ }^{1 *}$, Stefan Puchner ${ }^{2}$, Ashley M Lee ${ }^{2}$, Ting Liu², Godtfred Holmvang ${ }^{2}$, Udo Hoffmann², Suhny Abbara², Umesh Sharma ${ }^{1,2}$

From 18th Annual SCMR Scientific Sessions

Nice, France. 4-7 February 2015

\section{Background}

In patients with hemodynamically significant atrioventricular (AV) blocks of unknown etiology, cardiac MRI (CMR) with tissue characterization can provide incremental diagnostic information. Correlation of initial CMR findings with future events recorded by permanent pacemakers (PPM) can characterize the natural history of these serious and potentially life-threatening cardiac conditions.

\section{Methods}

We studied a total of 78 patients with complete heart block, high-grade second degree heart block and symptomatic bundle branch blocks. In all patients, initial cardiac work-up was non-revealing for potential etiology, and CMR with late gadolinium enhancement (LGE) was performed. Following CMR, most of these patients underwent clinically indicated PPM placement. The interrogated PPM events were followed up for 1-4 years

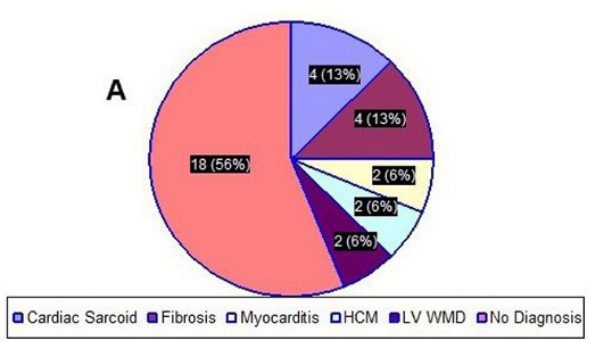

B

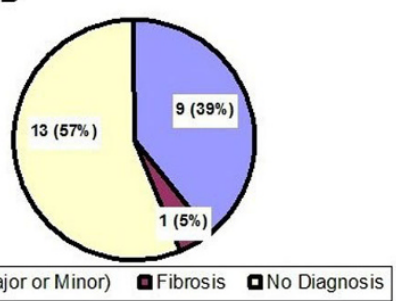

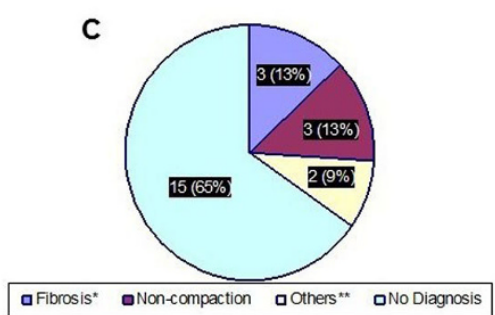

Figure 1 Pie Chart showing the relative distribution of the diagnostic categories of patients with- A) Complete or High-Grade AV-Block, B)RBBB, and C) LBBB.

${ }^{1}$ Internal Medicine, University at Buffalo, Buffalo, NY, USA

Full list of author information is available at the end of the article

(c) 2015 Carmona-Rubio et al; licensee BioMed Central Ltd. This is an Open Access article distributed under the terms of the Creative 


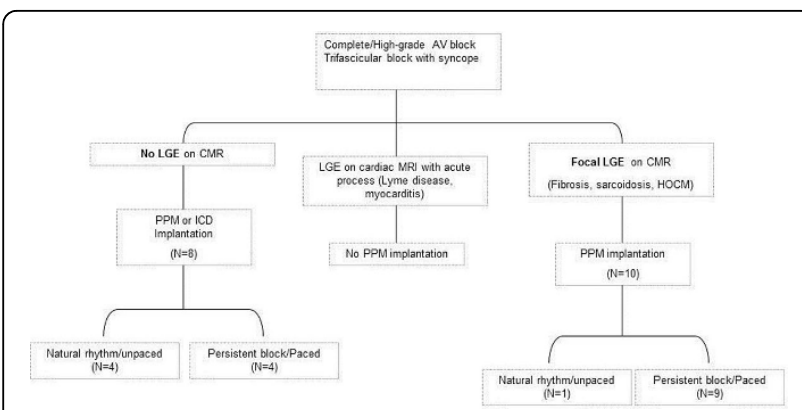

Figure 2 Pacemaker dependeance in patients with and without LGE or CMR.

to monitor significant arrhythmias or long-term pacemaker dependence.

\section{Results}

In patients with heart block, CMR identified a myocardial infiltrative process in $44 \%$. In patients with symptomatic LBBB or RBBB, CMR identified a plausible etiology in approximately $25 \%$. Presence of myocardial LGE by CMR had $90 \%$ sensitivity and $80 \%$ negative predictive value for long-term pacemaker dependence in patients with complete or high-grade AV blocks.

\section{Conclusions}

In addition to the indications attributed in the current diagnostic algorithm, CMR can identify potential cause of hemodynamically significant AV-blocks in over $40 \%$ of the patients. Presence of LGE or other major diagnostic findings on CMR can uniquely identify patients with long-term pacemaker dependence.

\section{Funding}

N/A.

\section{Authors' details}

${ }^{1}$ Internal Medicine, University at Buffalo, Buffalo, NY, USA. ${ }^{2}$ Cardiac CT/MRI/ PET Imaging, Department of Radiology, Massachusetts General Hospital, Boston, MA, USA.

Published: 3 February 2015

doi:10.1186/1532-429X-17-S1-P172

Cite this article as: Carmona-Rubio et al:: Cardiac magnetic resonance imaging and follow-up of pacemaker events to identify the etiology and natural history of heart blocks. Journal of Cardiovascular Magnetic Resonance 2015 17(Suppl 1):P172. 